\title{
Experience, Attitude, and Perceived Barriers Toward Research Among Ophthalmology Residents in Saudi Arabia: A National Cross-Sectional Study
}

\author{
Ali A Al Saeed (D)', Saad H AlEnezi $\mathbb{D}^{2}$, Mohanna Aljindan ${ }^{3}$, Fahad Alwadani ${ }^{4}$, Adi Mohammed Al Owaifeer ${ }^{4,5}$ \\ 'Collage of Medicine, King Faisal University, AIAhsa, Saudi Arabia; ${ }^{2}$ Department of Ophthalmology, College of Medicine, Imam Abdulrahman Bin Faisal \\ University, Dammam, Saudi Arabia; ${ }^{3}$ Department of Ophthalmology, Majmaah University, Al-Majmaah, Saudi Arabia; ${ }^{4}$ Faculty of Ophthalmology, \\ College of Medicine, King Faisal University, Al-Ahsa, Saudi Arabia; ${ }^{5}$ King Khaled Eye Specialist Hospital, Riyadh, Saudi Arabia \\ Correspondence: Adi Mohammed Al Owaifeer, Faculty of Ophthalmology, College of Medicine, King Faisal University, Al-Ahsa, Saudi Arabia, \\ Tel +966135895777, Fax+966/35800820, Email aalowaifeer@kfu.edu.sa
}

\begin{abstract}
Introduction: Research sets the foundation for evidence-based practice in medicine. Globally resident doctors in various specialties are facing major obstacles to accomplish high quality research projects. Understanding these obstacles may help residents achieve their maximum research potential. This current study was undertaken to document the experience, attitude, and perceived barriers toward research among Saudi ophthalmology residents.

Methodology: A specific questionnaire was developed and validated for the purpose of this study. The questionnaire was distributed online via email to actively enrolled residents in all five ophthalmology training programs in Saudi Arabia.

Results: Out of a total number of 193 ophthalmology residents in all five training programs, 147 responded to the questionnaire yielding a $76.1 \%$ response rate the mean age of participants was $27.6 \pm 1.8$ and the number of males and females was almost equal. The vast majority [96.4\%] have worked on at least one research project before starting residency training. Involvement was mainly in the phases of concept and design [72.5\%], proposal preparation [85.9\%], the three most frequent obstacles to conducting research projects for trainees were burden of other activities [4.27], lack of protected time for research [4.11] and too many regulations in obtaining ethical approval [3.67].

Discussion: Our current study shows that ophthalmology residents understand the importance of clinical research, but they are facing a considerable number of barriers toward accomplishing high-quality research projects. Findings of our study may help program directors to address these barriers and improve the incorporation of research along with clinical training in residency curricula.

Keywords: ophthalmology residents, medical education, research, evidence-based medicine
\end{abstract}

\section{Introduction}

Academic research and scholarly activities are of significant impact on the medical education of residents. The potential benefits of research to residents are several. Foremost, research activity provides residents with the necessary skills required to be good clinicians which in turn will result in high-quality patient care, it has been proofed that scholarly activities of residents boost up numerous skills such as critical appraisal and clinical reasoning. ${ }^{1,2}$ Additionally, prior research experience represents an added value to residents applying for future jobs or fellowship positions. ${ }^{3}$ Finally, highquality scholarly work supports residents by promoting them in conferences and publications in scientific journals. ${ }^{4}$

Despite a considerable number of publications in the scientific literature that sufficiently indicate the paramount importance of scholarly activity, there is evidence that residents' scholarly work continues to lag. ${ }^{5}$ Nowadays, in the prosperous era of evidence-based medicine scholar work and research activities are more relevant to ophthalmology residents to provide their patients with up to date evidence-based medical care. ${ }^{6}$ Previous data amply showed that residents are encountering many barriers to conducting research. The reported barriers differed amongst previously reported studies. For instance, it has been shown that personal factors [eg lack of time, interest, and research skills] 
represent the chief barriers among family medicine physicians. ${ }^{7-9}$ On the contrary, organizational barriers including culture, resources, environment, and logistics are reported as main barriers by integrative healthcare residents. ${ }^{10}$

Because of the competitive nature of admission to ophthalmology residency, trainees accepted into ophthalmology programs may have prior research experience that gave them an advantage over other applicants during the admission process. Additionally, due to the unique clinical environment of ophthalmology training, the barriers that ophthalmology residents perceive might be different than barriers reported by residents in other specialties. Therefore, our current study aims to evaluate the past research experience of Saudi ophthalmology residents as well as to explore their attitudes toward research and identify the potential barriers that hinder residents from achieving their full research potential.

\section{Methodology}

This is a cross-sectional study that targeted ophthalmology residents in all residency training programs in Saudi Arabia during the study period [from May 2020 to July 2020]. The study protocol was conducted in accordance with the ethical standards set by the World Medical Association Declaration of Helsinki. ${ }^{11}$ The research has been approved by King Faisal University College of Medicine Research Ethical Committee.

\section{Study Instrument "Questionnaire"}

A detailed questionnaire was developed and validated for the purpose of this study following five steps. First, the questionnaire items were drafted after reviewing the pertinent literature and accumulating questions from prior studies that were performed on the same topic. ${ }^{12-15}$ Following that, similar and duplicate items were removed. Then, a panel consisting of five experts with prior experience in the field were consulted to validate the content of the questionnaire for representativeness, relevance, and clarity. After content validation, cognitive interviews were performed with 17 participants, and items that were vague or misinterpreted were addressed. Finally, pilot testing was performed to ascertain the internal consistency of the questionnaire. The overall internal consistency (Cronbach's alpha) of the questionnaire was 0.7 .

The final questionnaire was structured into four sections. The first section collected demographic and professional characteristics [eg age, gender, marital status, year of residency, and training center], and the second section evaluated prior experience in research. The third and fourth sections respectively were on residents' attitudes and potential barriers toward research. Barriers and attitudes toward research were measured using a five-point Likert scale that ranged from "strongly disagree" to "strongly agree", with a neutral category in the middle.

\section{Data Collection}

The questionnaire was delivered in an electronic format using Google forms to all ophthalmology residents (total number of 193) in the five residency programs within Saudi Arabia. An email invitation was sent to all residents and those that did not respond within two weeks were gently reminded via a phone call. Participation in the study was voluntary and anonymous, as the questionnaire did not collect any identifiable data. A consent was displayed on the first page of the questionnaire and proceeding beyond that page indicated a participant's consent.

\section{Statistical Analysis}

Data were exported from Google forms into a spreadsheet for analysis. The final database did not contain any missing data points as all fields in the questionnaire were mandatory to fill. Statistical analysis was conducted using R [RStudio version 1.1.463 Mac, RStudio Inc., Boston, MA]. Categorical variables were reported as frequencies and percentages, whereas numerical variables were reported as mean \pm standard deviation. Barriers toward research were visualized into a divergent stacked bar chart using a specific data visualization software [Tableau, Tableau Software, Seattle, WA, USA]. 


\section{Results}

\section{Participants}

Out of a total number of 193 ophthalmology residents in all five training programs, 147 responded to the questionnaire yielding a $76.1 \%$ response rate. The mean age of participants was $27.6 \pm 1.8$ and the number of males and females was almost equal. Nearly half (44.2\%) of the participants were from the Riyadh residency training program, whereas the remainder were distributed among the four other programs. Detailed demographic data are shown in Table 1.

\section{Residents' Research Background and Experience}

Details of residents' prior research experience are shown in Table 2. The vast majority (96.4\%) have worked on at least one research project before starting residency training. Involvement was mainly in the phases of concept and design (72.5\%), proposal preparation (85.9\%), data collection (88\%), and manuscript writing (75.4\%). The majority (83.8\%) of respondents have worked on cross-sectional studies, whereas only a small fraction $(3.5 \%)$ were involved in randomized clinical trials. The vast majority $(82.4 \%)$ stated that they had contributed to a published research project.

Furthermore, $7.7 \%$ of the residents had published more than 5 research projects.

\section{Attitude Toward Research}

Table 3 summarizes the findings on participants' attitude toward research. The majority of respondents believed that research allows the advancement of scientific and medical knowledge and education (80.3\%), research develops critical thinking (78.9\%), and research is a valuable asset to a fellowship application (74.8\%). Interestingly, a notable minority of residents do not believe that research is an important component of residency and clinical training (11.6\%).

\section{Perceived Barriers}

The strongest perceived barrier (4.27) toward conducting research was the burden of other educational activities such as exams (Figure 1). The second most perceived barrier was the lack of protected time allotted for research (4.11). Interestingly, lack of proficiency in English and lack of interest were the lowest reported perceived barriers toward conducting a research project (2.88 and 2.97 respectively).

\section{Recommendations to Optimize the Research Environment}

Participants were also asked about their recommendations to optimize the research environment during training (Figure 2). Remarkably the vast majority agreed that supervisors need to be more aware and committed to the projects

Table I Demographics

\begin{tabular}{|l|c|c|}
\hline & $\mathbf{n}$ & $\%$ \\
\hline Sex & & \\
Male & 75 & $51 \%$ \\
Female & 72 & $49 \%$ \\
Marital Status & & \\
Married & 60 & $40.80 \%$ \\
Single & 87 & $59.20 \%$ \\
Training Level & & \\
RI & 46 & $31.30 \%$ \\
R2 & 27 & $18.40 \%$ \\
R3 & 37 & $25.20 \%$ \\
R4 & 37 & $25.20 \%$ \\
Region & & \\
Riyadh & 65 & $44.20 \%$ \\
Eastern Province & 37 & $25.20 \%$ \\
Western Province & 23 & $15.60 \%$ \\
\hline
\end{tabular}


Table 2 Previous Research Experience

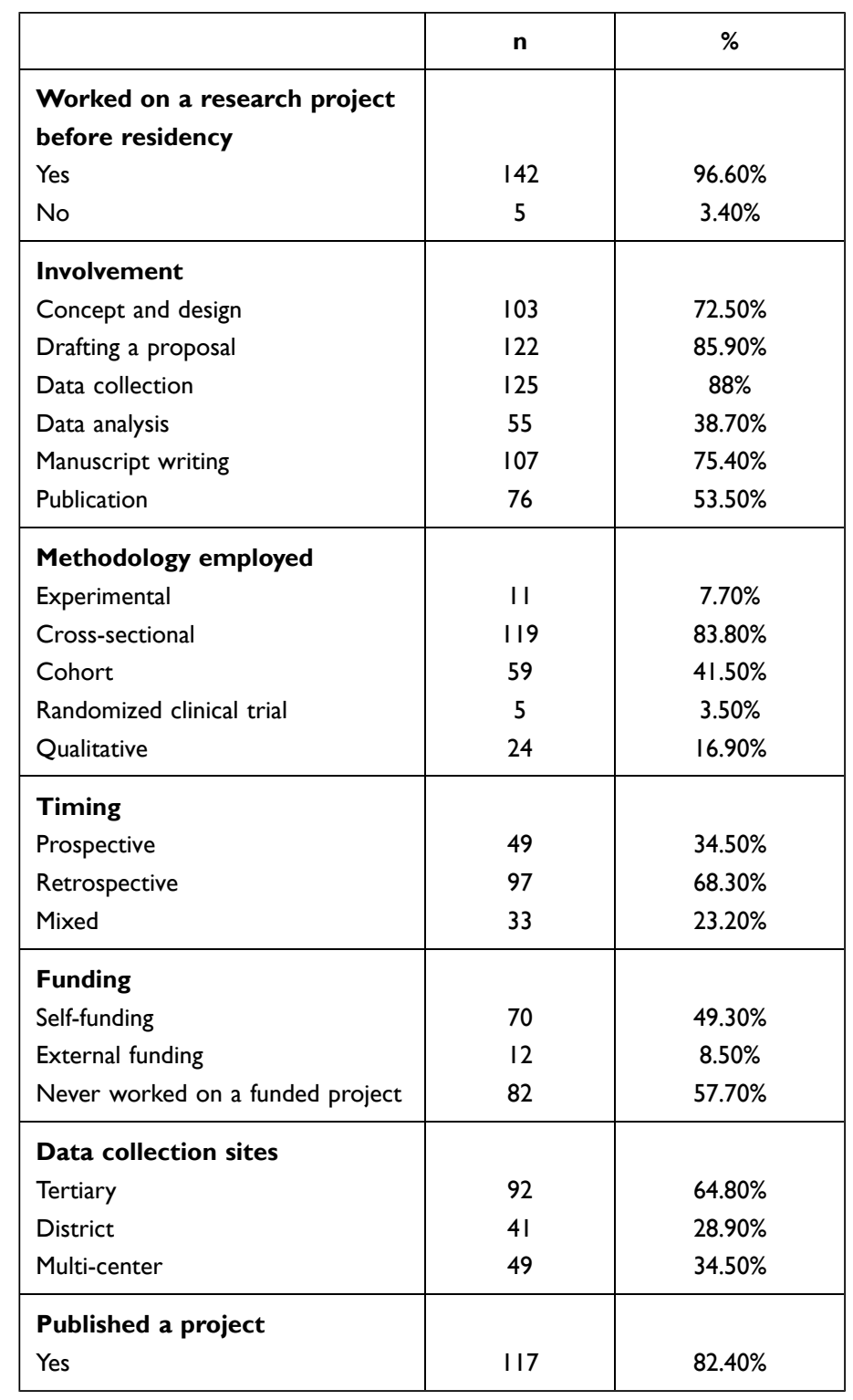

(81.6\%), more time needs to be given to residents for research (77.6\%), and that training centers should facilitate access to scientific journals $(66.7 \%)$. It is important to point out that only $15 \%$ agreed that research projects must be mandatory for promotion during residency.

Table 3 Attitude Toward Research

\begin{tabular}{|c|c|c|c|}
\hline Item & SA/A & $\mathbf{N}$ & SD/D \\
\hline Research is an asset to a fellowship position application & $74.80 \%$ & $15.00 \%$ & $8.80 \%$ \\
\hline Research is an important component of ophthalmology residency and clinical training & $73.50 \%$ & $21.10 \%$ & $11.60 \%$ \\
\hline Research improves clinical proficiency and quality of patient care & $72.10 \%$ & $17.00 \%$ & $6.80 \%$ \\
\hline Research develops essential skills for lifelong learning & $74.80 \%$ & $17.70 \%$ & $8.20 \%$ \\
\hline Research develops critical thinking & $78.90 \%$ & $16.30 \%$ & $3.40 \%$ \\
\hline Research allows the advancement of scientific/medical knowledge and education & $80.30 \%$ & $23.80 \%$ & $3.40 \%$ \\
\hline Research facilitates training of residents to be clinician investigators/scientists & $70.10 \%$ & $16.30 \%$ & $6.10 \%$ \\
\hline
\end{tabular}

Abbreviations: SA, strongly agree; A, Agree; N, neutral; SD, strongly disagree; D, disagree. 


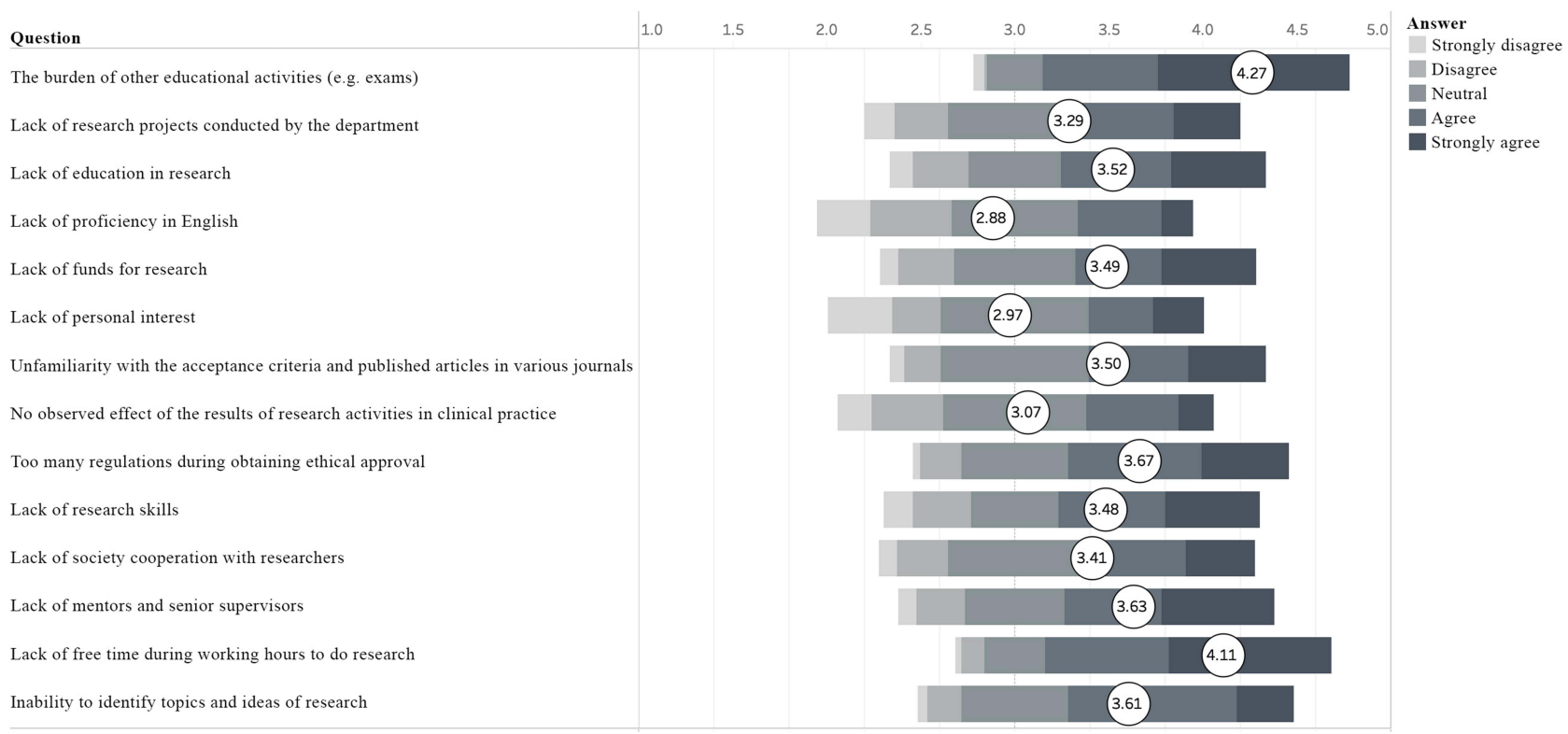

Figure I Perceived barriers.

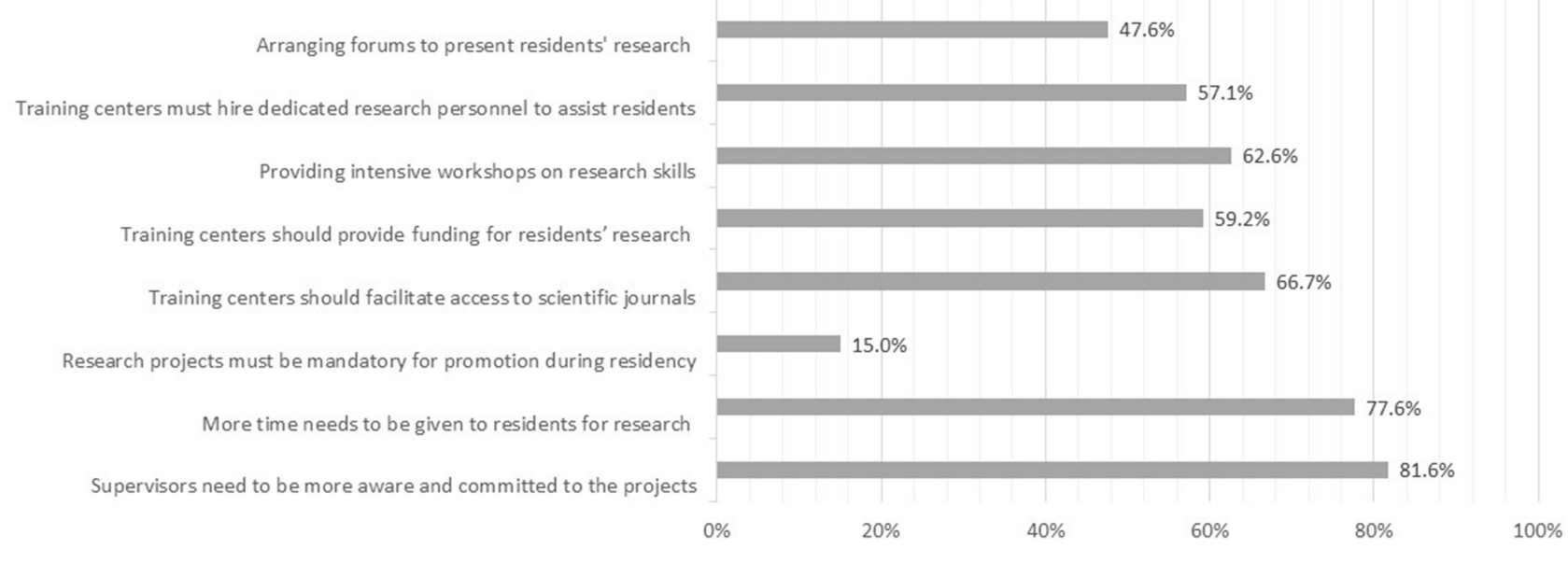

Figure 2 Recommendations to optimize the research environment.

\section{Discussion}

High-standard academic medical centers worldwide are encouraging residents and Faculty to engage in research. Yet, worldwide there is evidence that residents' research engagement continues to lag. ${ }^{5}$ Around the world, few studies have investigated residents' perspectives and perceived barriers to conducting research projects. ${ }^{16}$ Saudi Arabia is a rapidly developing country in the Middle East region with plenty of resources in comparison to other Middle East countries. Over the past couple of decades, Saudi Arabia has made remarkable efforts toward improving the level of education, health services, academia, and research. ${ }^{17}$ Therefore, it was of interest to investigate ophthalmology residents' research status and perspectives in Saudi Arabia. To our knowledge, this is the first national study surveying the barriers to research in ophthalmology residency in Saudi Arabia.

One of the most substantial factors that can motivate the initiation of any work is personal interest. The vast majority of the studied population amply demonstrated a passionate interest and positive attitude toward clinical research, with the 
majority agreeing that research enhances clinical knowledge and promotes critical thinking (Table 3), which is consistent with the findings of Kern et al. ${ }^{18}$

A considerable number of perceived barriers and the associated factors for efficiently conducting ophthalmic research by residents in Saudi Arabia have been identified. As illustrated in Figure 1, the burden of other educational activities (4.27) and lack of protected time allotted to research [4.11] are the top barriers toward conducting high-quality ophthalmic research. Moreover, around $77.6 \%$ of residents suggested that training programs must devote time for residents' research (Figure 2). The limitation of time seems to be a constant barrier for conducting research across different specialties, times, and regions around the world. ${ }^{19,20}$ Same barrier was perceived by Canadian plastic surgery residents. ${ }^{21}$ Like other surgical residency programs, ophthalmology residency program demands high workloads. Ophthalmology Residents have a total commitment to their surgical training might find difficulties to integrate research in their overloaded schedule.

A considerable number of participants agreed that the difficult and lengthy process of obtaining ethical approval represents a strong barrier to conducting research (3.67). It is professionally known that any clinical research involving human data utilization, patients' participation, or interventions to patients care must be reviewed by an ethical committee prior to initiating the project. All high-quality health care institutions have local or regional research ethics committees (REC), also known as institutional review boards (IRB). According to our data, IRB approval can be a significant obstacle in front of numerous investigators as reported in multiple studies. ${ }^{22}$ The steps of applying to and communicating with REC/IRB can be extremely complex and troublesome for investigators, especially in the presence of other demotivating factors such as the limitation of time and lack of funds. At the end of our questionnaire, we included an optional empty box for residents to add any further comments they had. One resident mentioned that "IRB should not complicate things" another commented by saying "too much regulation are demotivating for starting any novel idea".

As reported in the previous literature, investigators usually have difficulty in both steps of application and communication with IRB/REC. In a study published by Ito-Ihara et $a{ }^{23},{ }^{23}$ the percentage of doctors at Kyoto and Seoul University Hospitals reporting difficulties in communicating with IRB/REC was $68 \%$ and $24 \%$ respectively. There is a genuine need for carefully reviewing these administrative bureaucracies that might hinder the conduction of research projects. Hiring a professional research personnel can help in assessing residents to avoid such a difficulties in obtaining IRB Approval.

It is not reassuring to note that lack of mentors and senior supervisors was the fourth most cited barrier by residents (3.62). Furthermore, $81.6 \%$ of the respondents suggested that supervisors need to be more aware and committed to the projects. Undoubtedly, mentoring positively correlates with the achievement of mentee outcomes. ${ }^{24}$ Residency training programs should be structured in a manner that encourages mentors and senior supervisors to pass their own experience to the next generation and actively supervise residents' research projects.

Nowadays, health institutions are acknowledging the paramount importance of mentorship programs, thereby they are formalizing the process of mentorship by assigning each junior resident with a senior supervisor formal mentoring), however, some mentees prefer to find someone they respect and trust to help them reach their objectives [Informal mentoring]. ${ }^{25}$ Either formal or informal mentoring can be effective. Once the partnership is underway, both mentors and mentees must sustain this relationship to produce the best out of it.

Difficulty in selecting an appropriate topic and the lack of education about the research process were two other perceived barrier that residents in our study agreed with (3.61 and 3.52 respectively). Besides adequate mentoring of residents, curricula of training programs must also include formal teaching on research methodology, fundamentals of biostatistics, and manuscript writing, as such approaches will ensure that residents are capable of producing high-quality research projects. ${ }^{1}$

\section{Limitation}

This is a study that targeted Saudi ophthalmology residents, the generalization of our results to the rest of the world is not possible, therefore we recommend further research that covers wider geographic areas outside Saudi Arabia. 


\section{Conclusions}

Our results showed that Saudi ophthalmology residents are interested in scholar work and clinical research, however, the lack of dedicated time, complex research approval processes, and financial funding limitations are frequent challenges. A strategy to face the current challenges is necessary to improve residents' research productivity. This strategy must focus on devoting time for residents' research, hiring dedicated research personnel, providing intensive research training courses, and ensuring active mentoring for residents.

\section{Abbreviations}

IRB, Institutional review board; REC, Research ethics committee; SA, strongly agree; A, Agree; N, Neutral; SD, Strongly disagree; $\mathrm{D}$, disagree.

\section{Data Sharing Statement}

The datasets generated and/or analysed for this study isn't publicly available, permission must be obtained from KFUIRB after contacting the corresponding author.

\section{Ethical Approval and Consent to Participate}

Participation of the Residents was on a voluntary basis. No personal information was collected. Informed consent obtained from the participants before enrollment in the study. The study protocol was conducted in accordance with the ethical standards set by the World Medical Association Declaration of Helsinki. ${ }^{11}$ Ethical approval was obtained from the Ethics Committee of collage of medicine, King Faisal University (KFU).

\section{Acknowledgment}

The manuscript has been deposited as a preprint and include any associated DOI:10.21203/rs.3.rs-111601/v1.

\section{Author Contributions}

All authors made a significant contribution to the work reported, whether that is in the conception, study design, execution, acquisition of data, analysis and interpretation, or in all these areas; took part in drafting, revising or critically reviewing the article; gave final approval of the version to be published; have agreed on the journal to which the article has been submitted; and agree to be accountable for all aspects of the work.

\section{Funding}

This project did not receive any financial funding.

\section{Disclosure}

All authors declare no conflicts of interest.

\section{References}

1. Abramson M. Improving resident education: what does resident research really have to offer? Trans Am Acad Ophthalmol Otolaryngol. 1977;84:984-985.

2. Does research make for better doctors? Lancet. 1993;342(8879):1063-1064. doi:10.1016/0140-6736(93)92055-X

3. Souba WW, Tanabe KK, Gadd MA, Smith BL, Bushman MS. Attitudes and opinions toward surgical research. A survey of surgical residents and their chairpersons. Ann Surg. 1996;223(4):377-383. doi:10.1097/00000658-199604000-00006

4. Fournier I, Stephenson K, Fakhry N, et al. Barriers to research among residents in otolaryngology - head \& neck surgery around the world. Eur Ann Otorhinolaryngol Head Neck Dis. 2019;136(3):S3-S7.

5. Jain MK, Cheung VG, Utz PJ, Kobilka BK, Yamada T, Lefkowitz R. Saving the endangered physician-scientist — a plan for accelerating medical breakthroughs. N Engl J Med. 2019;381(5):399-402. PMID: 31365796. doi:10.1056/NEJMp1904482

6. Kohlwes RJ, Shunk RL, Avins A, et al; The PRIME curriculum. Clinical research training during residency. J Gen Intern Med. 2006;21:506-509. doi:10.1111/j.1525-1497.2006.00438.x

7. Bammeke F, Liddy C, Hogel M, Archibald D, Chaar Z, MacLaren R. Family medicine residents' barriers to conducting scholarly work. Can Fam Physician. 2015;61:780-787. 
8. Gill S, Levin A, Djurdjev O, Yoshida EM. Obstacles to residents' conducting research and predictors of publication. Acad Med. 2001;76:477. doi:10.1097/00001888-200105000-00021

9. Mitwalli H, Al Ghamdi K, Moussa N. Perceptions, attitudes, and practices towards research among resident physicians in training in Saudi Arabia. EMHJ. 2014;20:99-104.

10. Verhoef MJ, Mulkins A, Kania A, Findlay-Reece B, Mior S. Identifying the barriers to conducting outcomes research in integrative health care clinic settings - a qualitative study. BMC Health Serv Res. 2010;10:14. doi:10.1186/1472-6963-10-14

11. World Medical Association. World Medical Association declaration of Helsinki: ethical principles for medical research involving human subjects. JAMA. 2013;310(20):2191-2194. PMID: 24141714. doi:10.1001/jama.2013.281053

12. Canadian Plastic Surgery Research Collaborative [CPSRC]. Barriers and attitudes to research among residents in plastic and reconstructive surgery: a National Multicenter Cross-Sectional Study. J Surg Educ. 2017;74(6):1094-1104. PMID: 28551364. doi:10.1016/j.jsurg.2017.04.004

13. Barzkar F, Baradaran HR, Koohpayehzadeh J. Knowledge, attitudes and practice of physicians toward evidence-based medicine: a systematic review. J Evid Based Med. 2018;11(4):246-251. PMID: 30430759. doi:10.1111/jebm.12325

14. Olaussen A, Jennings PA, O’Reilly G, Mitra B, Cameron PA. Barriers to conducting research: a survey of trainees in emergency medicine. Emerg Med Australas. 2017;29(2):204-209. PMID: 28097829. doi:10.1111/1742-6723.12734

15. Teh LC, Prema M, Choy MP, Letchuman GR. Attitudes, barriers and facilitators to the conduct of research in government hospitals: a cross-sectional study among specialists in government hospitals, northern states of Malaysia. Med J Malaysia. 2017;72(1):26-31. PMID: 28255136.

16. Chan JY, Narasimhalu K, Goh O, et al. Resident research: why some do and others don't. Singapore Med J. 2017;58(4):212-217. doi:10.11622/ smedj.2016059

17. Deleu D, Northway MG, Hanssens Y. Geographical distribution of biomedical publications from the Gulf corporation council countries. Saudi Med J. 2001;22(1):10-12.

18. Kern DC, Parrino TA, Korst DR. The lasting value of clinical skills. JAMA. 1985;254:70-76. doi:10.1001/jama.1985.03360010076030

19. Sabzwari S, Kauser S, Khuwaja AK. Experiences, attitudes and barriers towards research amongst junior faculty of Pakistani medical universities. BMC Med Educ. 2009;9(1):68. doi:10.1186/1472-6920-9-68

20. Lloyd T, Phillips BR, Aber RC, et al. Factors that influence doctors' participation in clinical research. Med Edu. 2004;38(8):848. doi:10.1111/ j.1365-2929.2004.01895.x

21. Ross S, Grant A, Counsell C, Gillespie W, Russell I, Prescott R. Barriers to participation in randomised controlled trials: a systematic review. J Clin Epidemiol. 1999;52(12):1143-1156. doi:10.1016/S0895-4356(99)00141-9

22. English RA, Lebovitz Y, Giffin RB. Institute of Medicine (US) Forum on Drug Discovery, Development, and Translation. Transforming Clinical Research in the United States: Challenges and Opportunities: Workshop Summary. Washington (DC): National Academies Press; 2010.

23. Ito-Ihara T, Hong J-H, Kim O-J, et al. An international survey of physicians regarding clinical trials: a comparison between Kyoto University Hospital and Seoul National University Hospital. BMC Med Res Methodol. 2013;13(1):130. doi:10.1186/1471-2288-13-130

24. Eby LT, Allen TD, Evans SC, Ng T, Dubois D. Does mentoring matter? A multidis-ciplinary meta-analysis comparing mentored and non-mentored individuals. J Vocat Behav. 2008;72(2):254-267.

25. Straus SE, Chatur F, Taylor M. Issues in the mentor-mentee relationship in academic medicine: a qualitative study. Acad Med. 2009;84(1):135-139. doi:10.1097/ACM.0b013e31819301ab

Clinical Ophthalmology

\section{Publish your work in this journal}

Clinical Ophthalmology is an international, peer-reviewed journal covering all subspecialties within ophthalmology. Key topics include: Optometry; Visual science; Pharmacology and drug therapy in eye diseases; Basic Sciences; Primary and Secondary eye care; Patient Safety and Quality of Care Improvements. This journal is indexed on PubMed Central and CAS, and is the official journal of The Society of Clinical Ophthalmology (SCO). The manuscript management system is completely online and includes a very quick and fair peer-review system, which is all easy to use. Visit http://www. dovepress.com/testimonials.php to read real quotes from published authors.

Submit your manuscript here: https://www.dovepress.com/clinical-ophthalmology-journal 